\title{
Dietary fat intake and blood pressure in 10 to 19 years adolescents in the United Kingdom
}

\author{
Z. Li, C. Evans and J. Cade \\ Nutritional Epidemiology Group, School of Food Science and Nutrition, University of Leeds, LS2 9JT, UK
}

Studies supported that total fat intake was associated with increased risks of hypertension, but as a subgroup of fat, n-3 polyunsaturated fatty acid (PUFA) intake had association with reduced risk of hypertension ${ }^{(1)}$. Relevant studies in adolescents are lacking, although the prevalence of hypertension among adolescents is high and is increasing ${ }^{(2)}$, and elevated blood pressure in youth is a strong predictor of hypertension in adulthood ${ }^{(3)}$. The aim of this study is to determine the relationship between dietary fat intake and blood pressure in 10-19 years UK adolescents.

A sample of 585 10-19 years old adolescents were selected from the UK National Diet and Nutrient Survey in which participants were drawn randomly. General information, anthropometric and blood pressure (BP) were collected, and dietary data was assessed from a four-day food record. BP data was transformed to systolic BP Z-scores (SBPZ) and diastolic BP Z-scores (DBPZ) ${ }^{(4)}$. Univariable and multivariable linear regression was undertaken for BP/BPZ-scores). Age, gender, weight, height, alcohol intake, smoking, sodium intake and energy intake were adjusted in models.

Rate of hypertensive participants was $7.2 \%$. Every $1 \mathrm{~g}$ increase in n3-PUFA intake was associated with $3.49 \mathrm{mmHg}$ decreased DBP ( $95 \% \mathrm{CI}:-6.86$ to $-0.12, \mathrm{p}=0.042)$, but not related with SBP or Z-scores. Every $1 \mathrm{~g}$ polyunsaturated low fat spread intake was related with $0.86 \mathrm{mmHg}(95 \% \mathrm{CI}:-1.61$ to $-0.12, \mathrm{p}=0.024)$ reduced DBP and $0.035(95 \% \mathrm{CI}:-0.06$ to $-0.01, p=0.019)$ reduced DBPZ, but not with SBP or SBPZ. No statistically significant association was found between total/other subgroup of fat intake and blood pressure values/Z-scores.

\begin{tabular}{|c|c|c|c|c|}
\hline \multirow[b]{2}{*}{ Nutrients } & \multicolumn{4}{|c|}{ Change in BP per unit change in exposure } \\
\hline & Coefficient & $95 \% \mathrm{CI}$ & & $\mathrm{P}$ value $(* \mathrm{p}<0.05)$ \\
\hline \multicolumn{5}{|l|}{ With systolic blood pressure } \\
\hline Low fat spread not polyunsaturated $(\mathrm{g})$ & $0 \cdot 045$ & $-2 \cdot 295$ & $2 \cdot 385$ & 0.97 \\
\hline Low fat spread polyunsaturated (g) & $-1 \cdot 281$ & $-2 \cdot 566$ & $0 \cdot 004$ & $0 \cdot 051$ \\
\hline Cis $n-3$ fatty acids $(\mathrm{g})$ & -5.434 & $-11 \cdot 256$ & $0 \cdot 387$ & $0 \cdot 067$ \\
\hline Cis $n-6$ fatty acids (g) & -1.456 & $-3 \cdot 018$ & $0 \cdot 105$ & $0 \cdot 067$ \\
\hline \multicolumn{5}{|l|}{ With diastolic blood pressure } \\
\hline Low fat spread not polyunsaturated $(\mathrm{g})$ & $0 \cdot 174$ & $-1 \cdot 179$ & $1 \cdot 528$ & $0 \cdot 801$ \\
\hline Low fat spread polyunsaturated $(\mathrm{g})$ & $-0 \cdot 858$ & -1.601 & $-0 \cdot 115$ & $0.024^{*}$ \\
\hline Cis $n-3$ fatty acids (g) & $-3 \cdot 488$ & $-6 \cdot 855$ & $-0 \cdot 121$ & $0.042 *$ \\
\hline Cis n-6 fatty acids (g) & -0.783 & -1.687 & $0 \cdot 121$ & 0.089 \\
\hline \multicolumn{5}{|l|}{ With systolic blood pressure Z-scores } \\
\hline Low fat spread not polyunsaturated (g) & $0 \cdot 003$ & -0.065 & $0 \cdot 07$ & 0.94 \\
\hline Low fat spread polyunsaturated $(\mathrm{g})$ & $0 \cdot 002$ & -0.02 & $0 \cdot 025$ & $0 \cdot 843$ \\
\hline Cis $n-3$ fatty acids $(\mathrm{g})$ & -0.072 & $-0 \cdot 196$ & $0 \cdot 052$ & $0 \cdot 255$ \\
\hline Cis $n-6$ fatty acids (g) & $-0 \cdot 01$ & $-0 \cdot 041$ & $0 \cdot 021$ & $0 \cdot 523$ \\
\hline \multicolumn{5}{|l|}{ With diastolic blood pressure $Z$-scores } \\
\hline Low fat spread not polyunsaturated (g) & $0 \cdot 023$ & $-0 \cdot 047$ & 0.093 & $0 \cdot 517$ \\
\hline Low fat spread polyunsaturated $(\mathrm{g})$ & $-0 \cdot 035$ & $-0 \cdot 064$ & $-0 \cdot 006$ & $0.019^{*}$ \\
\hline Cis $n-3$ fatty acids (g) & $-0 \cdot 107$ & $-0 \cdot 227$ & $0 \cdot 013$ & $0 \cdot 079$ \\
\hline Cis $\mathrm{n}-6$ fatty acids $(\mathrm{g})$ & -0.008 & -0.038 & 0.022 & $0 \cdot 616$ \\
\hline
\end{tabular}

In NDNS adolescent population, dietary n3-PUFA intake was associated with reduced DBP, and dietary polyunsaturated low fat spread intake was associated with DBP and DBPZ.

1. Cabo J, Alonso R, Mata P (2012) Br J Nutr 107 Suppl 2, S195-200.

2. Muntner P, He J, Cutler JA et al. (2004) JAMA 291, 2107-2113.

3. Sun SS, Grave GD, Siervogel RM et al. (2007) Pediatrics 119, 237-246.

4. NHBPEP Working Group (2004) Pediatrics 114, 555-76. 